\title{
THE DEGREES OF THE FACTORS OF CERTAIN POLYNOMIALS OVER FINITE FIELDS
}

\author{
W. H. MILLS
}

\begin{abstract}
Neal Zierler has discovered that the polynomial $x^{585}+x+1$ over GF(2) is the product of 13 irreducible factors of degree 45 and that the polynomial $x^{16513}+x+1$ over $G F(2)$ is the product of 337 irreducible factors of degree 49 . We prove a general theorem that includes these results, as well as some other well known results, as special cases.
\end{abstract}

Let $K$ be a finite field containing exactly $q$ elements. Let $r$ be a power of $q$, say $r=q^{n}$. For any polynomial $f(x)=\sum a_{i} x^{i}$ over $K$ we set

$$
\hat{f}(x)=\sum a_{i} x^{(r i-1) /(r-1)}
$$

and

$$
\hat{f}^{\beta}(x)=x f\left(x^{r-1}\right)=\sum a_{\mathfrak{i}} x^{r i} .
$$

Lemma 1 (ORE). Let $A(x)$ and $B(x)$ be polynomials over $K$ and set $C(x)=A(x) B(x)$. Then $C^{\beta}(x)=A^{\beta}\left(B^{\beta}(x)\right)$.

Proof. Set $A(x)=\sum a_{i} x^{i}$ and $B(x)=\sum b_{j} x^{j}$. Then

$$
\begin{aligned}
A^{\beta}\left(B^{\beta}(x)\right) & =\sum_{i} a_{i}\left(\sum_{j} b_{j} x^{r j}\right)^{r i} \\
& =\sum_{i, j} a_{i} b_{j} x^{i+j} \\
& =C^{\beta}(x) .
\end{aligned}
$$

Theorem 1. Let $f(x)$ and $g(x)$ be polynomials over $K$. Then $f(x) \mid g(x)$ if and only if $\hat{f}(x) \mid \hat{g}(x)$.

Proof. Suppose first that $f(x) \mid g(x)$ and set $g(x)=h(x) f(x)$. By Lemma 1 we have $g^{\beta}(x)=h^{\beta}\left(f^{\beta}(x)\right)$. Since $x \mid h^{\beta}(x)$ this gives us $f^{\beta}(x) \mid g^{\beta}(x)$. Therefore we have $\hat{f}\left(x^{r-1}\right) \mid \hat{g}\left(x^{r-1}\right)$ which implies that $\hat{f}(x) \mid \hat{g}(x)$.

On the other hand suppose that $\hat{f}(x) \mid \hat{g}(x)$ and set $g(x)=A(x)+B(x)$, where $f(x) \mid A(x)$ and the degree of $B(x)$ is less than that of $f(x)$. By the first part of the proof we have $\hat{f}(x) \mid \hat{A}(x)$ so that

Received by the editors October 27, 1969.

AMS Subject Classifications. Primary 1225.

Key Words and Phrases. Factors of polynomials, polynomials over finite fields. 


$$
0 \equiv \hat{g}(x)=A(x)+\hat{B}(x) \equiv \hat{B}(x) \quad(\bmod \hat{f}(x)) .
$$

Now the degree of $\hat{B}(x)$ is less than that of $\hat{f}(x)$, so that $\hat{B}(x)=0$ and $B(x)=0$. Therefore we have $f(x) \mid g(x)$.

THEOREM 2. Suppose $f(x) \mid x^{N}-1$ and let $d$ be a factor of $r-1$. Then the degree of every irreducible factor of $\hat{f}\left(x^{d}\right)$ over $K$ divides $n N$.

Proof. By Theorem 1 we have $\hat{f}(x) \mid x^{\left(r^{N}-1\right) /(r-1)}-1$. This is equivalent to $\hat{f}\left(x^{d}\right) \mid x^{d\left(r^{N}-1\right) /(r-1)}-1$. Since $d \mid r-1$ this implies that $\hat{f}\left(x^{d}\right) \mid x^{r^{N}-1}$ -1 . Therefore every root of $\hat{f}\left(x^{d}\right)$ lies in $\mathrm{GF}\left(r^{N}\right)$. Since $r^{N}=q^{n N}$ this implies that the degree of every irreducible factor of $\hat{f}\left(x^{d}\right)$ over $K$ divides $n N$.

CoRollary. If $r=q^{n}$, then the degree of every irreducible factor of $x^{1+r}+x+1$ over $\mathrm{GF}(q)$ divides $3 n$.

This corollary is the special case of Theorem 2 with $f(x)=x^{2}+x+1$, $N=3$, and $d=1$. It is well known and proofs have been given by a number of authors. See $[1$, p. 93].

Using Theorem 2 we can obtain many other results of the same nature. For example, since $x^{3}+x+1$ divides $x^{7}-1$ over $\mathrm{GF}(2)$ we see that if $r=2^{n}$, then the degree of every irreducible factor of $x^{1+r+r^{2}}+x+1$ over GF(2) divides $7 n$.

Similarly if $r=2^{n}$, then the degree of every irreducible factor of $x^{1+r+r^{2}+r^{3}}+x+1$ over GF(2) divides $15 n$.

When certain additional conditions are satisfied the degrees of the irreducible factors of $\hat{f}\left(x^{d}\right)$ are all equal to $n N$. To show this we need the following result.

Lemma 2. Let $f(x)$ be an irreducible polynomial over $K$, and let $g(x)$ be an arbitrary polynomial over $K$. Suppose for some positive integer $d, \hat{f}\left(x^{d}\right)$ and $\hat{g}\left(x^{d}\right)$ have a root in common. Then $f(x) \mid g(x)$.

Proof. Let $h(x)$ be the greatest common divisor of $\hat{f}\left(x^{d}\right)$ and $\hat{g}\left(x^{d}\right)$. Then $h(x)$ is not a constant. Let $\mathfrak{a}$ be the set of all polynomials $A(x)$ over $K$ such that $h(x) \mid \hat{A}\left(x^{d}\right)$. Using Theorem 1 we see that $\mathfrak{a}$ is an ideal in the principal ideal ring $K[x]$. Since $f(x) \in \mathfrak{a}, 1 \notin \mathfrak{a}$, and $f(x)$ is irreducible, it follows that a consists of precisely the multiples of $f(x)$. Since $g(x) \in \mathfrak{a}$, we have $f(x) \mid g(x)$ and the proof is complete.

Theorem 1 and Lemma 2 are closely related to results of Zierler [3].

THEOREM 3. Let $f(x)$ be an irreducible polynomial over $K$ with period $N$. Let $d$ be a factor of $r-1$ and set $r-1=$ de. Suppose that $(e, d N)=1$ and that every prime factor of $n$ is also a factor of $N$. Then every irreducible factor of $\hat{f}\left(x^{d}\right)$ over $K$ has degree $n N$. 
Proof. Since $f(x) \mid x^{N}-1$ it follows from Theorem 1 that

$$
\hat{f}(x) \mid x^{(r N-1) /(r-1)}-1 .
$$

Replacing $x$ by $x^{d}$ we obtain $\hat{f}\left(x^{d}\right) \mid x^{\left(r^{N}-1\right) / e}-1$. Let $\alpha$ be a root of $\hat{f}\left(x^{d}\right)$. Then we have $\alpha^{\left(r^{N}-1\right) / e}=1$ and $\alpha \in \mathrm{GF}\left(r^{N}\right)$. Now $r \equiv 1(\bmod e)$ and therefore

$$
\begin{aligned}
\left(r^{N}-1\right) / e & =d\left(r^{N}-1\right) /(r-1) \\
& =d\left(r^{N-1}+r^{N-2}+\cdots+r+1\right) \\
& \equiv d N \quad(\bmod e) .
\end{aligned}
$$

Since $(e, d N)=1$ it follows that $e$ is relatively prime to the order of $\alpha$. Let $m$ be the degree of $\alpha$ over GF $(r)$. Then $m \mid N$ and $\alpha^{r^{m}-1}=1$. Since $e$ is relatively prime to the order of $\alpha$ we have

$$
1=\alpha^{\left(r^{m}-1\right) / e}=\alpha^{d\left(r^{m}-1\right) /(r-1)} .
$$

This gives us $\hat{B}\left(\alpha^{d}\right)=0$ where $B(x)=x^{m}-1$. Thus $\hat{f}\left(x^{d}\right)$ and $\hat{B}\left(x^{d}\right)$ have a root in common. By Lemma 2 we have $f(x) \mid B(x)$. Since $N$ is the period of $f(x)$ this gives us $N \mid m$, and therefore $m=N$.

Now let $M$ be the degree of $\alpha$ over $K$. Then $M \mid n N$. Suppose $M$ $<n N$. Then for some prime $\lambda$ we have $\lambda M \mid n N$. Since every prime factor of $n$ is also a factor of $N$ we have $\lambda \mid N$. Thus $\alpha$ is contained in a field of degree $n(N / \lambda)$ over $K$. This field has degree $N / \lambda$ over $\mathrm{GF}(r)$, which implies that $m<N$, a contradiction. Therefore we have $M=n N$. Since $\alpha$ was an arbitrary root of $\hat{f}\left(x^{d}\right)$ it follows that every irreducible factor of $\hat{f}\left(x^{d}\right)$ over $K$ has degree $n N$, and the proof is complete.

Setting $d=r-1$ and $e=n=1$ in Theorem 3 we obtain the following result:

CoRollary 1. (Zierler's generalization of the theorem of Ore, Gleason, and Marsh.) Let $f(x)$ be an irreducible polynomial over $\mathrm{GF}(q)$, say $f(x)=\sum a_{i} x^{i}$. Let $N$ be the period of $f(x)$. Then every irreducible factor of $\sum a_{i} x^{q^{i}-1}$ over $\mathrm{GF}(q)$ has degree $N$.

We observe that $x^{2}+x+1$ is irreducible over $\mathrm{GF}(q)$ if and only if $q \equiv 2(\bmod 3)$. Thus setting $d=1, n=3^{s}, f(x)=x^{2}+x+1$, and $N=3$ we obtain the following special case of Theorem 3 :

Corollary 2. If $q \equiv 2(\bmod 3), n=3^{s} \geqq 1, r=q^{n}$, and $d=1$, then every irreducible factor of $x^{1+r}+x+1$ over $\mathrm{GF}(q)$ has degree $3 n$.

Setting $q=2, n=7^{8}, d=1, f(x)=x^{3}+x+1$, and $N=7$ in Theorem 3 we obtain the following result: 
CoROLlary 3. If $n=7^{s} \geqq 1$ and $r=2^{n}$, then the degree of every irreducible factor of $x^{1+r+r^{2}}+x+1$ over GF(2) is $7 n$.

For example, $x^{16513}+x+1$ is the product of 337 irreducible factors over GF(2), each of which has degree 49.

Similarly, setting $q=2, n=3^{8} 5^{t}, d=1, f(x)=x^{4}+x+1$, and $N=15$ we obtain this result:

COROLlaRy 4. If $n=3^{8} 5^{t} \geqq 1$ and $r=2^{n}$, then every irreducible factor of $x^{1+r+r^{2}+r^{3}}+x+1$ over $\mathrm{GF}(2)$ has degree $15 n$.

For example, $x^{585}+x+1$ is the product of 13 irreducible factors of degree 45 over GF(2).

\section{REFERENCES}

1. Solomon W. Golomb, Shift register sequences, Holden-Day, San Francisco, Calif., 1967.

2. Oystein Ore, Contributions to the theory of finite fields, Trans. Amer. Math. Soc. 36 (1934), 243-274.

3. Neal Zierler, On the theorem of Gleason and Marsh, Proc. Amer. Math. Soc. 9 (1958), 236-237.

Institute for Defense Analyses, Princeton, New Jersey 08540 